\title{
Characterising Mineral Slurry Dewatering through Laboratory Centrifugation
}

\author{
$\underline{\text { S. Berres }}^{\text {a }}$, R. Garcés ${ }^{\text {a }}$, and S.P. Usher ${ }^{b}$ \\ ${ }^{a}$ Departamento de Ciencias Matemáticas y Físicas, Facultad de Ingeniería, \\ Universidad Católica de Temuco, Chile \\ ${ }^{b}$ Particulate Fluids Processing Centre, Department of Chemical and Biomolecular Engineering, \\ The University of Melbourne, Victoria, 3010, Australia \\ Email: sberres@uct.cl
}

\begin{abstract}
Water plays a key role in the mining industry, mainly in supporting exploration and mineral extraction. Cost efficiency can be obtained by minimising the use of water. Efficient methods of water use minimisation during the recovery process are suggested to build on a framework for modelling and simulation of mine water use during dewatering. Dewatering can be described by a one-dimensional model for a thickener, centrifuge or pressure filter, where the local solids concentration depends on time and position.
\end{abstract}

The dewatering dynamics is described by two material-specific model functions of the local solids concentration $\phi$ : The hindered settling function $R(\phi)$ quantifies or hydrodynamic resistance to flow of liquid through the slurry. This resistance is mathematically related to a solids settling flux function $f(\phi)$. When all particles are in contact, i.e. when the local particle concentration is greater than the gel point, the slurry forms a continuous network and has a network strength that can transmit stress. The compressive yield stress $P_{y}(\phi)$ or effective solids stress function $\sigma_{e}(\phi)$ quantifies the strength of the particle network in compression, with the strength being zero at the gel point $\phi_{g}$ or critical concentration $\phi_{c}$.

In order to obtain a scalable model, the dewatering model can be calibrated on laboratory scale with help of a laboratory centrifuge and an algorithm for parameter identification. In centrifugation, the tailings slurry is described by its local solids concentration (volume fraction) $\phi=\phi(r, t)$ as a function of radial position $r$ and time $t$. As a model simplification, it is assumed that the sludge has a relatively homogeneous composition, i.e. it is composed of uniform solid particles having same material properties like an effective average diameter and density. A slurry that only consists of one single type of particles is referred to as a "monodisperse" suspension. The settling of a monodisperse flocculated suspension in a rotating tube centrifuge can be modelled by the following spatially one-dimensional partial differential equation (PDE)

$$
\frac{\partial \phi}{\partial t}+\frac{\partial}{\partial r}\left(-\frac{\omega^{2} r}{g} f(\phi)\right)=\frac{\partial^{2} A(\phi)}{\partial r^{2}}
$$

where $\omega$ is the angular velocity, $g$ is the acceleration of gravity, the batch hindered settling function $f(\phi)$ is the first of two material-specific model functions of the local solids concentration $\phi$, and $A(\phi)$ is a diffusion function depending on both $f(\phi)$ and $\sigma_{e}(\phi)$.

Two applications of this PDE earlier described by Berres et al. (2010) and Usher et al. (2013) are compared for their utility in determining material properties for process modelling. For the first method, the constitutive functions are given in parametric form; the parameters are identified by solving an inverse problem through an optimisation routine. The gel point turns out to be a highly sensitive parameter which induces a natural illposedness. By the second method, the raw measurement data is used to characterise material property values at a number of solids concentrations. These data can then be fitted to determine parameters for constitutive functions. Both methods deliver functions for the phenomenological description of mine water dewatering.

Keywords: Dewatering, gel point, Centrifugation, Numerical modelling, Sedimentation, Compression, Flocculated Suspension, Parameter Identification, Sensitivity Analysis 


\section{INTRODUCTION}

Water plays a key role in the mining industry, mainly in supporting exploration and mineral extraction. Cost efficiency can be obtained by minimising the use of water. Efficient methods of water use minimisation during the recovery process are suggested to build on a framework for modelling and simulation of mine water use during dewatering. Dewatering can be described by a one-dimensional model for a thickener (Usher (2005), Bürger et al. (2004)), centrifuge (Bürger and Concha (2001)) or pressure filter (Landman and White (1997), Bürger et al. (2001)), where the local solids concentration depends on time and position.

The dewatering dynamics is described by two material-specific model functions of the local solids concentration $\phi$. The hindered settling function $R(\phi)$ quantifies or hydrodynamic resistance to flow of liquid through the slurry. This resistance is mathematically related to the settling velocity by the relation

$$
u(\phi)=\frac{\Delta \rho g(1-\phi)^{2}}{R(\phi)},
$$

and a solids settling flux function

$$
f(\phi)=\phi u(\phi)
$$

where $\Delta \rho$ is the solid-liquid density difference and $g$ is gravitational acceleration. A common parametric forms for the flux function $f(\phi)$ is described by Richardson and Zaki (1954):

$$
f(\phi)= \begin{cases}v_{\infty} \phi(1-\phi)^{C} & \text { for } 0 \leq \phi \leq 1, \\ 0 & \text { otherwise, }\end{cases}
$$

with the parameters $v_{\infty}<0$, corresponding to the settling velocity of a single particle in an unbounded fluid, and the Richardson-Zaki exponent $C>1$.

When all particles are in contact, i.e. when the local particle concentration is greater than the gel point, the slurry forms a continuous network and has a network strength that can transmit stress (Buscall and White (1987)). The compressive yield stress $P_{y}(\phi)$ or effective solids stress function $\sigma_{e}(\phi)$ quantifies the strength of the particle network in compression, with the strength being zero at the gel point $\phi_{g}$ or critical concentration $\phi_{c}$. The power law of Tiller and Leu (1980) gives a typical expression for the effective solid stress function,

$$
\sigma_{e}(\phi)=\sigma_{0}\left(\left(\frac{\phi}{\phi_{c}}\right)^{k}-1\right) \text {, for } \phi>\phi_{c} \text { or } 0 \text { otherwise, }
$$

with parameters $k>0, \sigma_{0} \geq 0$ and $0<\phi_{\mathrm{c}} \leq 1$. A derived parameter is termed the solids diffusivity function

$$
D(\phi)=P_{y}{ }^{\prime}(\phi) \frac{(1-\phi)^{2}}{R(\phi)}, \quad \text { where } P_{y}{ }^{\prime}(\phi)=\frac{d P_{y}(\phi)}{d \phi},
$$

which is analogously defined as the diffusion coefficient

$$
a(\phi)=\sigma_{e}{ }^{\prime}(\phi) \frac{f(\phi)}{\Delta \rho g \phi}, \quad \text { where } \sigma_{e}^{\prime}(\phi)=\frac{d \sigma_{e}(\phi)}{d \phi},
$$

where the integrated diffusion function $A(\phi)$ is given by

$$
A(\phi):=\int_{0}^{\phi} a(s) \mathrm{d} s,
$$

The settling of a monodisperse flocculated suspension in a rotating tube centrifuge can be modelled by the following spatially one-dimensional partial differential equation (PDE)

$$
\frac{\partial \phi}{\partial t}+\frac{\partial}{\partial r}\left(-\frac{\omega^{2} r}{g} f(\phi)\right)=\frac{\partial^{2} A(\phi)}{\partial r^{2}}
$$

where $\omega$ is the angular velocity. Equation (8), together with suitable initial and boundary conditions, can be discretised by a fully implicit first-order finite difference scheme to allow the efficient simulation of the settling process. The initial suspension solids volume fraction $\phi_{0}=\phi(r, t=0)$ and the initial suspension height $h_{0}=r_{0}-r_{\max }$, is defined relative to the initial radius $r_{0}$ and maximum radius at the base of the centrifuge sample $r_{\max }$. 
The model employed herein is based on the assumption that there exists a critical concentration (or gel point) denoted by $\phi_{c}$, such that wherever $\phi=\phi(r, t)<\phi_{c}$, there is no contact between the particles, while for $\phi=\phi(r, t) \geq \phi_{\mathrm{c}}$, the particles form a compressible sediment layer. This means that

$$
\sigma_{\mathrm{e}}{ }^{\prime}(\phi) \begin{cases}=0 & \text { for } \phi \leq \phi_{\mathrm{c}}, \\ >0 & \text { for } \phi>\phi_{\mathrm{c}} .\end{cases}
$$

For details on the underlying sedimentation-consolidation theory we refer to Berres et al. (2003). In mathematical terms, we obtain that the governing PDE (8) is of second-order parabolic type for $\phi>\phi_{c}$. For $\phi<\phi_{\mathrm{c}}$, Equation (8) degenerates into the first-order hyperbolic equation of a kinematic centrifugation model. The basic problem is that the location of the type-change interface $\phi=\phi_{c}$, corresponding to the sediment level, is not known a priori and is part of the solution.

The phenomenological theory supports a framework for an accurate description of the dewatering of mineral slurries. The present contribution addresses the problem of identifying certain material specific parameters that define the functions $R(\phi)$ or $f(\phi)$ and $P_{y}(\phi)$ or $\sigma_{e}(\phi)$ from measured concentration profiles. Two different methods, a curve fit optimisation method described by Berres et al. (2010), and an approximation described by Usher et al. (2013) are compared for their utility in determining material properties for process modelling.

\section{OPTIMISATION METHOD}

In what follows, we will refer to concentration profiles that have been obtained from measurements and, alternatively, by the (numerical) solution of the mathematical model for a given choice of these parameters, as observed and simulated data, respectively. The inverse problem of parameter identification consists in varying the (unknown) parameters until the best approximation of the observation by the simulation is achieved. The degree of approximation, that is, the distance between observed and simulated data, is defined by a suitable cost function. Consequently, the problem of parameter identification can be regarded as the optimisation problem of minimising this cost function. The variation of parameters is done herein by a QuasiNewton method. Sensitivity indicators like the condition number of the Hessian matrix of the cost function in its minimum are calculated.

In the present model one can distinguish three different types of parameters: the so-called "parabolic" parameters appear in the function $\sigma_{e}(\phi)$ and therefore affect only the (degenerating) diffusion function; the "hyperbolic" parameters are those of the function $f(\phi)$ and appear in both the convective and diffusive terms; and the critical concentration $\phi_{c}$ as a "hyperbolic-parabolic switch" parameter that controls whether the diffusion function is active, i.e., the mixture is subject to sediment compressibility at $(r, t)$.

The number of identifiable parameters is usually restricted by the quantity of information in the observation. For example, if there is only one single datum of information (e.g., the speed supernatantsuspension interface at one given time), which corresponds to the flux function, then only one parameter in $f(\phi)$ can be identified, since there is only one equation to solve. For a given non-linear parametric form, some parameters are supposed to have a stronger independent influence on the solution structure, whereas other parameters are more correlated. One goal of this study is to identify different degrees of independence of parameters in our sedimentation-consolidation model. Therefore, the influence of various parameter subsets is compared. One central goal is to identify those parameters, which have the strongest correlation and exclude them from the set of parameters to identify. The goal is to obtain an appropriate parameter set, where the parameters are meaningful in the sense that they show only small correlations with each other.

The optimisation method makes use of the numerical method to solve the direct problem, which consists of an initial-boundary value problem for (1) that describes the centrifugal settling process. The space-time domain is discretised by a grid with spatial cells of width $\Delta r=\left(R_{2}-R_{1}\right) / M$, where $\mathrm{M}$ is an integer, and a time step $\Delta t=T / N$ such that there are points $r_{j}=R_{1}+j \Delta r, t_{n}=n \Delta t, j=0, \ldots, M, n=1, \ldots, N$, for which the approximation $\phi_{j}^{n} \approx \phi\left(r_{j}, t_{n}\right)$ is computed, starting from $\phi_{j}^{0}=\phi_{0}$. The marching formula in the interior has the form 
Berres et al., Characterising mineral slurry dewatering through laboratory centrifugation

$$
\frac{\phi_{j}^{n+1}-\phi_{j}^{n}}{\Delta t}-\frac{\omega^{2}}{\Delta r}\left(r_{j+1 / 2} f_{j+1 / 2}^{n+1}-r_{j-1 / 2} f_{j-1 / 2}^{n+1}\right)=\frac{A\left(\phi_{j+1}^{n+1}\right)-2 A\left(\phi_{j}^{n+1}\right)+A\left(\phi_{j-1}^{n+1}\right)}{(\Delta r)^{2}},
$$

whereas at the domain boundaries there are wall conditions.

\section{APPROXIMATE METHOD:}

The approximate method described by Usher et al. (2013) characterises material properties over a constrained range of solids concentrations from transient and equilibrium centrifugation test data. A key feature of these approximations is that they produce data that is not reliant on predefining constitutive equations for the material properties a priori. However, the results do need to be combined with data at higher solids concentrations, e.g. pressure filtration (Usher et al. (2001)) to produce comprehensive curve fits over a wide range of solids concentrations.

Transient sediment interface height data at early times, where $\phi$ is nominally unnetworked $\left(\phi<\phi_{c}\right)$, is inverted to produce $R(\phi)$ data at solids concentrations ranging from the initial solids concentration to the gel point and sometimes even higher solids concentration with reasonable accuracy. The method is conceptually similar to Kynch batch settling analysis (Kynch (1952)) adapted centrifugal coordinates. A number of evenly distributed solids volume fraction values, $\phi_{1}$, are selected over the range $\left(\phi_{0}, \phi_{a v}\right)$, where $\phi_{a v}$ is the average solids volume fraction at the end of the test such that

$$
\phi_{a v}=\frac{\phi_{0} h_{0}}{h_{f}}
$$

with $h_{f}$ being the final sediment-liquid interface height. For each $\phi_{i}$, a mass balance,

$$
h_{i}(0)=\frac{\phi_{0} h_{0}}{\phi_{i}},
$$

is used to calculate the equivalent initial height, $h_{i}(0)$, and for every point on the settling curve, $h(t)$, the value of $u\left(\phi_{i}\right)$ is determined using

$$
u\left(\phi_{i}\right)=\frac{g}{\omega^{2} t} \ln \left(\frac{r_{\max }-h(t)}{r_{\max }-h_{i}(0)}\right),
$$

with the maximum observed value of $u\left(\phi_{i}\right)$ being selected. All of the $u\left(\phi_{i}\right)$ data points are converted to $R\left(\phi_{i}\right)$ data points using Eq. (1) or $f\left(\phi_{l}\right)$ using Eq. (2). The output data is truncated subject to a novel criterion that rejects data at higher solids concentrations that do not satisfy the following:

$$
\frac{d}{d \phi}\left(\frac{d \ln [R(\phi)]}{d \phi}\right)<0
$$

A series of equilibrium test height data, $h_{e q}$, at a range of rotation speeds are used to determine a corresponding series of $P_{y}(\phi)$ data points using an algorithm first proposed by Buscall and White (1987),

$$
\phi_{\text {base }}=\frac{\phi_{0} h_{0}\left[1-\frac{1}{2 r_{\max }}\left(h_{e q}+a \frac{d h_{e q}}{d a}\right)\right]}{\left(h_{e q}+a \frac{d h_{e q}}{d a}\right)\left(1-\frac{h_{e q}}{r_{\max }}\right)+\frac{h_{e q}{ }^{2}}{2 r_{\max }}}
$$

and

$$
P_{y}\left(\phi_{\text {base }}\right)=\Delta \rho a \phi_{0} h_{0}\left(1-\frac{h_{e q}}{r_{\max }}\right),
$$

where the acceleration, $a=\omega^{2} r_{\max }$ and $\phi_{\text {base }}=\phi\left(r_{\max }, t \rightarrow \infty\right)$ is the solids volume fraction at the base of the suspension at equilibrium. This is achieved with a curve fit of $h_{e q}$ vs. $a$. 
Berres et al., Characterising mineral slurry dewatering through laboratory centrifugation

\section{RESULTS}

As a test case the parameters of Bürger and Concha (2001) are chosen. There, the density difference is $\Delta \rho=$ $1660 \mathrm{~kg} \mathrm{~m}^{-3}$ and the parameters for the flux function $f(\phi)$ and the effective solids stress $\sigma_{e}(\phi)$ are

$$
v_{\infty}=-0.0001, C=5.0, \phi_{c}=0.1, \sigma_{0}=5.7, \mathrm{k}=9.0 .
$$

For a standard test, the angular velocity is $\omega=327 \mathrm{rad} \mathrm{s}^{-1}(3122.62 \mathrm{rpm})$ such that $\omega^{2} r_{\max }=10000 \mathrm{~g}$, with $r_{\max }=0.3 \mathrm{~m}$. The initial conditions $\left(\phi_{0}, h_{0}\right)$, are chosen as $(0.07,0.24)$.

A key criterion of the suitability of the choice of the PDE model is the sensitivity of the parameter set. This sensitivity depends on the shape of the cost function that indicates the difference of the model prediction to the observed data. In Figure 2 (left) the cost function for a parameter set consisting of the pair $\left(C, \phi_{c}\right)$ and in Figure 1 (right) the evolution of the cost function depending on the parameter set $\left(C, \phi_{c}\right)$ during the identification process with different initial guesses are shown. The cost function is obtained with intermediate observation profiles. It can be seen that the cost functions are is non-convex. Convexity, at least close to the minimum however is a requirement that optimisation schemes work.

A quantitative assessment of the cost function can be done by inspection of its Hessian matrix. The condition numbers of the Hessian matrices matrix in the cost minimum indicates whether the chosen parameter set is highly sensitive and therefore should be reduced; a high sensitivity corresponds to a big condition number. If the parameter set contains all four parameters then the condition numbers are calculated as $\kappa\left(H_{i}\right)=7.7643 \cdot 10^{4}$ and $\kappa\left(H_{s}\right)=1.2847 \cdot 10^{8}$ for the cases of intermediate and stationary observation profiles, respectively. The intermediate data delivers a condition number roughly 'double as good' as the stationary data. Looking at the entries of the Hessian matrices it can be observed that the entry corresponding to the second derivative with respect to the critical concentration, $J_{u_{c} u_{c}}$, is dominant by several orders of magnitude, i.e., all the whole matrix is dominated by that single term. Simultaneously, the other parameters correlate negatively with the critical concentration $u_{c}$. Observing the diagonals of the Hessian matrices one can judge that the parameter $u_{c}$ is distinctly most sensitive, followed by $\sigma_{0}$ and then $k$ whereas parameter $c$ is least sensitive. Comparing the condition numbers of the Hessian matrix in the cost optimum for all parameter sets with two or three parameters confirms this observed hierarchy of parameter sensitivity.

This study of the condition number can be refined by looking at the distribution of the singular values of the Hessian matrix for different subsets of parameter sets. Among the three-parameter-sets the set $(C, \sigma, k)$ has the most equidistributed singular values, indicating that this triple represents a "good choice" with respect to parameter sensitivity; see Berres et al. (2010) for more details.
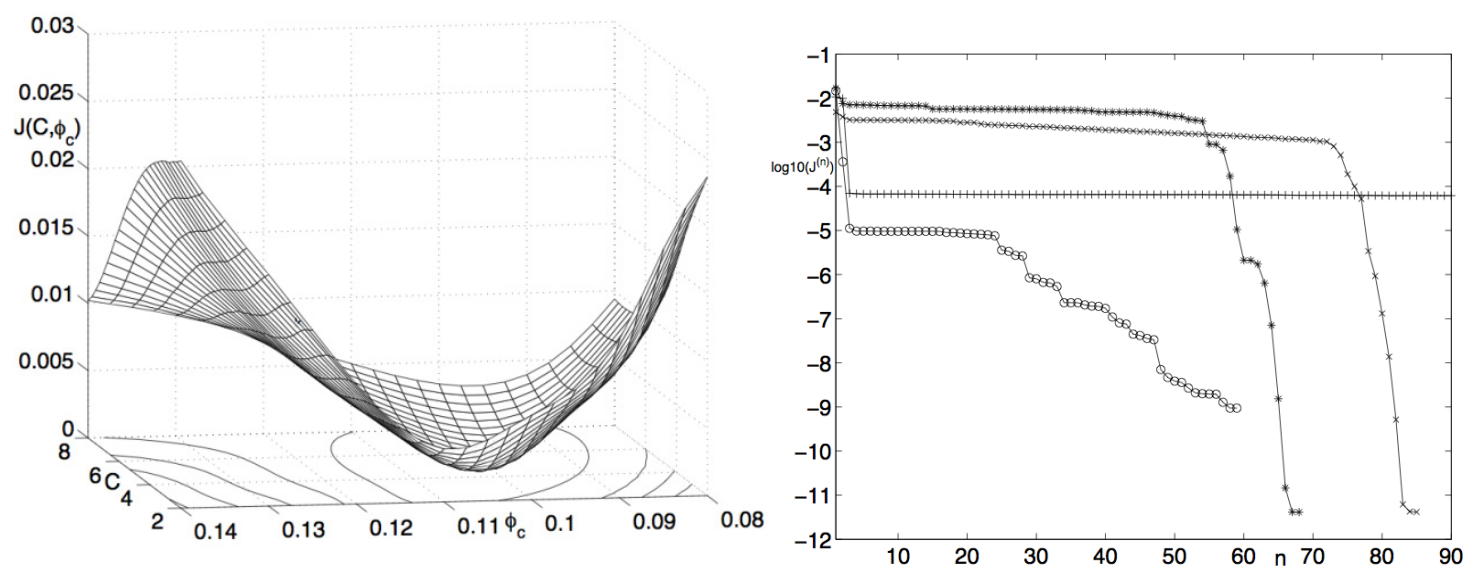

Figure 1. Two-parameter cost functions for each parameter set $\left(C, \phi_{c}\right)$ for the intermediate observation profile (left). Evolution of the cost function depending on the parameter set $\left(C, \phi_{c}\right)$ during the identification process with different initial guesses (right).

Alternatively, using the approximation methods to determine the material properties produces data points as shown in Figure 3. Determination of network strength, $P_{y}(\phi)$, is achieved by centrifugation to equilibrium over a number of rotation speeds, 50-400 rpm and 500-4000 rpm, with the excellent results shown in Figure 3. Results show that the fit is excellent, however, they do not cover the full range of solids concentrations, 
which could be extended by conducting the equilibrium tests over a wider range of rotation rates. This method can be applied to confirm whether the functional form being used to fit $P_{y}(\phi)$ is appropriate.

The determination of settling velocity or hindered settling function, $R(\phi)$, behaviour requires transient test data as shown in Figure 2 for $\phi_{0}=0.07$ and $0.20 \mathrm{v} / \mathrm{v}$ at $\omega=327 \mathrm{rad} \mathrm{s}^{-1}(3122.62 \mathrm{rpm})$. The approximation method relies on there being a region analogous to the fan region often observed in batch settling. Unfortunately, this region does not exist in this case and instead there is a shock from free settling to an almost fully compressed network bed. However, the data has been analysed and the results shown in Figure 2b. With $\phi_{0}$ being to the left of the peak in the flux curve, the amount of accurate permeability data produced is limited. The method breaks down because of the significant reduction in solids concentration at the suspension liquid interface at early times, before this interface becomes networked. See Figure 4 in Burger and Concha (2001) for details. The method requires modification to account for this behaviour. These results suggest that in the absence of a fan region, the approximation method can only produce an estimate of the settling rate behaviour. However, it should be noted that the model functional forms for the material properties described by Bürger and Concha (2001) and used here are not typical of flocculated mineral slurries usually containing fine particles which can exhibit different centrifugation behaviour.

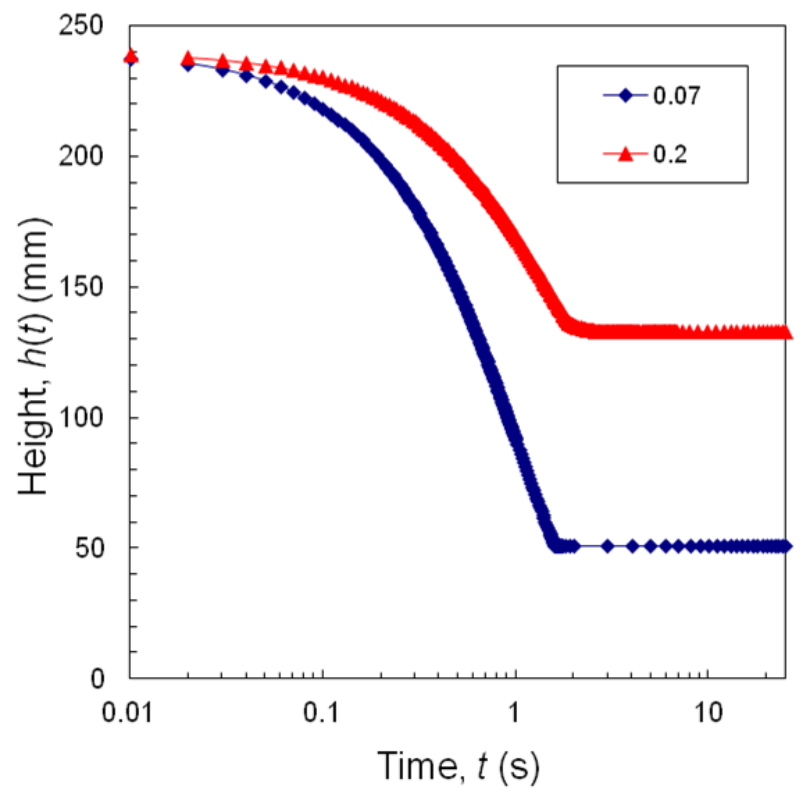

Figure 2. Transient centrifugation suspension-liquid interface height data with $\phi_{0}=0.07$ and $0.20 \mathrm{v} / \mathrm{v}$.
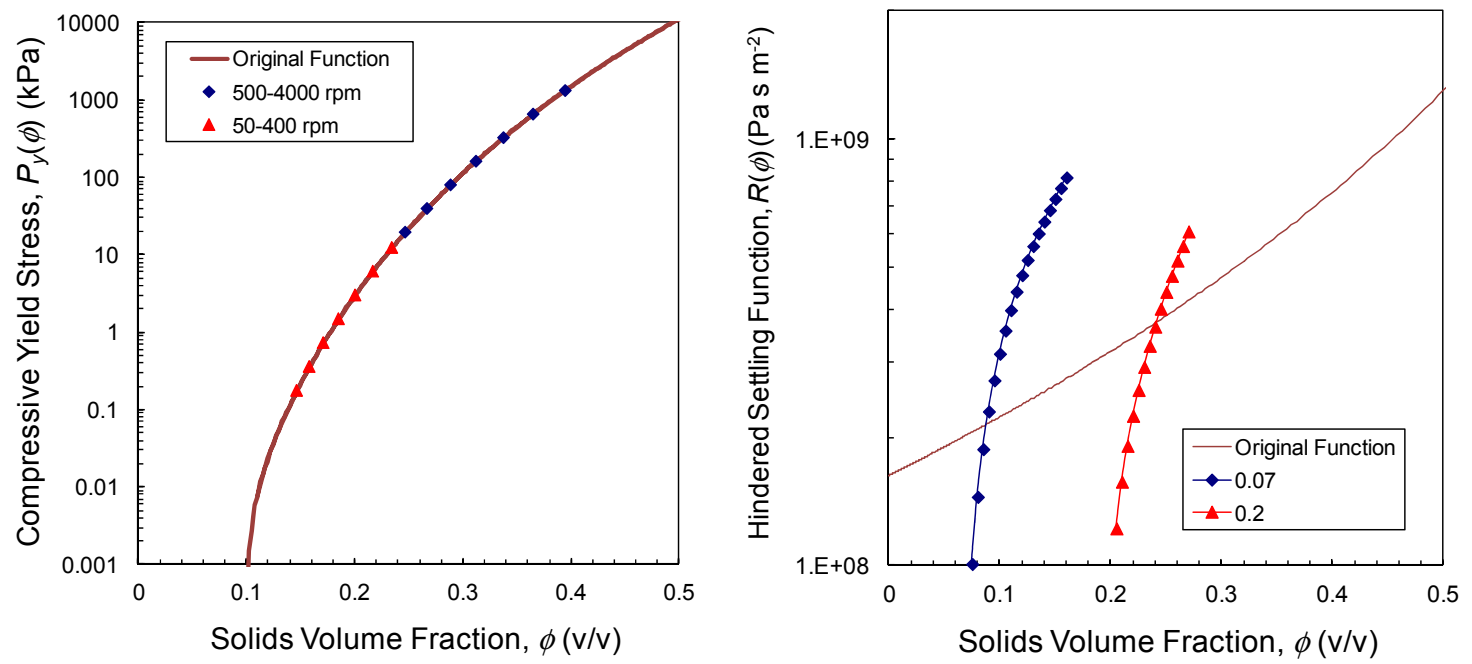

Figure 3. Approximation method analysis results compared to original functional forms from Bürger and Concha (2001). 


\section{DISCUSSION AND CONCLUSIONS}

Whereas in previous studies on parameter identifications of flocculated suspensions at several instances the ill-posedness has been observed, here the convergence behaviour has been evaluated quantitatively. In particular, the two cases, where observation data in form of intermediate and stationary profile, are considered and compared. As a central result, of the study of the Hessian matrices, the intermediate data provide parameter identification, which is by a factor 2 (on a logarithmic scale) more robust and reliable than with stationary data. In previous studies it was detected that the critical concentration plays a crucial role in the ill-posedness of the overall identification, but this observation has not been pursued further. Here, parameter sets with and without the critical concentration are evaluated.

As a surprising result the identification parameter set containing all three parameters that determine the form of the effective solids stress provides for stationary data remarkable worse identification behaviour than the other cases that include the critical concentration. This is surprising since in the case of intermediate profile the set of the three diffusive parameters does not behave such bad even though then the parabolic parameters do not play an active role.

The ill-posedness far from the cost minimum is illustrated qualitatively by the plots of the cost functions both for one-parameter and two-parameter sets. There one can observe non-convexity in various instances like convex-concave regions and saddle-point structure by negative combined second derivatives. This nonconvexity contradicts all assumptions on gradient methods like the cg or the Quasi-Newton method, which ideally intend an approximation by a quadratic positive definite function

A series of identifications is performed with varying parameter sets and initial guesses far from the cost minimum. Several parameter choices, which provide very slow or no convergence are detected and this inconvenient behaviour is documented together with the condition number of the Hessian matrix. By these test identifications the feasibility of the parameter identification in dependence of the choice of the parameter sets indicated by the study before is confirmed.

As a complement to the optimisation method, the approximation method can provide a very good initial estimate of the material properties being determined and also provide insights into what constitutive forms are most appropriate for the optimisation methods described.

\section{ACKNOWLEDGMENTS}

SB acknowledges support by Conicyt (Chile) through Fondecyt project 1120587.

\section{REFERENCES}

Berres, S. and R. Bürger (2003). On gravity and centrifugal settling of polydisperse suspensions forming compressible sediments. Int. J. Sol. Struct. 40, 4965-4987.

Berres S., R. Bürger, and R. Garcés (2010). Centrifugal settling of flocculated suspensions: A sensitivity analysis of parametric model functions. Dry. Tech. 28, 858-870.

Bürger, R. and F. Concha (2001). Settling velocities of particulate systems 12: batch centrifugation of flocculated suspensions. Int. J. Min. Proc. 63, 115-145.

Bürger, R., F. Concha, and K.H. Karlsen (2001). Phenomenological model of filtration processes: 1. Cake formation and expression. Chem. Eng. Sci. 56, 4537-4553

Bürger, R., J.J.R. Damasceno, and K.H. Karlsen (2004). A mathematical model for batch and continuous thickening of flocculated suspensions in vessels with varying cross-section. Int. J. Min. Proc. 73, 183-208.

Buscall, R. and L.R. White (1987). The consolidation of concentrated suspensions. J. Chem. Soc. Faraday Trans. I 83, 873-891.

Landman, K.A. and L.R. White (1997). Predicting filtration time and maximizing throughput in a pressure filter. AIChE J. 43, 3147-3160.

Richardson, J.F. and W.N. Zaki (1954). Sedimentation and fluidization: Part I. Trans. Inst. Chem. Eng. (London). 32, 35-53.

Tiller, F.M. and W.F. Leu (1980). Basic data fitting in filtration. J. Chin. Inst. Chem. Eng., 11, 61-70.

Usher, S.P., R.G. de Kretser, and P.J. Scales (2001). Validation of a new filtration technique for dewaterability characterization. AIChE J. 47, 1561-1570.

Usher S.P. and P.J. Scales (2005). Steady state thickener modelling from the compressive yield stress and hindered settling function. Chem. Eng. J. 111, 253-261.

Usher S.P., Studer L.J., Wall R.C., and Scales P.J. (2013), Characterisation of dewaterability from equilibrium and transient centrifugation test data. Chem. Eng. Sci. 93, 277-291. 